\title{
A Predictive and Battery Protective Control Strategy for Series HEV
}

\author{
Mir Hesam Hajimiri ${ }^{1}$ and Farzad Rajaei Salmasi ${ }^{2}$ \\ ${ }^{1}$ Department of Electrical and Computer Engineering, University of Tehran, h.hajimiri@gmail.com \\ ${ }^{2}$ Department of Electrical and Computer Engineering, University of Tehran,farzad_rs@ieee.org
}

\begin{abstract}
Hybrid Electric Vehicles (HEV) are superior to conventional vehicles from the standpoint of environmental issues. Many factors involve in designing HEVs such as fuel consumption, emission and performance. A major challenge for development of hybrid vehicles is coordination of multiple energy sources and converters, and in case of a HEV, power flow control for both mechanical and electrical path. This necessitates the utilization of appropriate control or energy management strategy. Furthermore, the durability extension of some critical components in the drive train such as batteries tends to be one of the substantial factors considered in designing control strategies for HEVs as replacement costs is a deterring factor for consumers. This paper proposes an improved power follower control strategy for series hybrid electric vehicles based on protection of the vehicle's battery and prediction of the future vehicles' path. First, a fuzzy predictive algorithm is integrated into a conventional power follower management system such that the future path information of the vehicle is taken into account for generation of the control signals. Then, the energy management system is augmented with a new tool to increase the state of the health (SOH) of the power train battery. Furthermore, since Valve Regulated Lead Acid (VRLA) batteries are of great importance in HEV technology, a new method based is used to optimize the charging current for these batteries, in order to decrease charging time and improve battery lifetime. This approach, which results in the extension of the battery life, is called Predictive and Protective Algorithm (PPA). The simulation results verify the effectiveness of the proposed controllers.
\end{abstract}

\section{Keywords}

hybrid electric vehicle, fuzzy logic controller, global positioning system, the state of health

\section{INTRODUCTION}

Seagoing vessels, heavy trucks, transition buses, and locomotives, are good candidates for hybrid vehicles with series power train. In the series structure, since the engine is mechanically separated from the axle, as shown in Figure 1, the engine/generator can be often operated in the low emission or high efficiency region based on the corresponding torque-speed characteristics (Miller, 2004). Nevertheless, we should remember that the failure of electric vehicles in the market was mainly due to

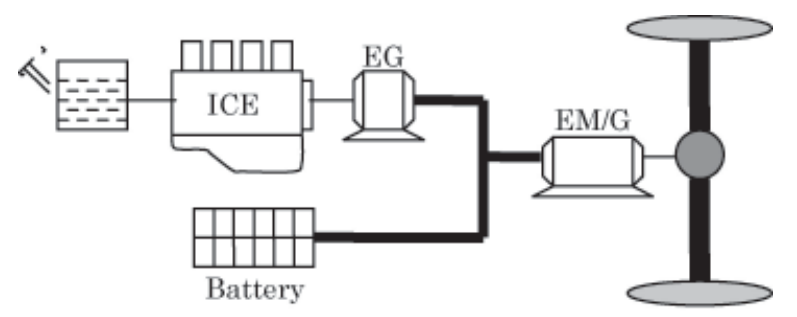

ICE: Internal combustion engine

EG: Electric generator

EM/G: Electric motor generator

Fig. 1 Series hybrid electric structure the failure of deeply discharged batteries in a couple of months. This problem can be extended to hybrid drive trains too, in which the battery play a main rule in the traction system (Chan, 2002). Consequently, a good energy management strategy should take care of emission reduction along with prolonging of components lives (Salmasi, 2007). Generally, an energy management or control strategy, which is usually implemented in the vehicle central controller, inputs the measurements of the vehicle operating conditions such as speed or acceleration, requested torque by the driver, current roadway type or traffic information. The outputs of a control strategy are decisions to turn on or off certain components, or to modify their operating regions by commanding local component controllers. (Ehsani et al., 2005) So far, there have been two general trends, dealing with the energy management problem in hybrid vehicles: rulebased, fuzzy or deterministic (Schouten et al., 2002) and optimization-based solutions (Delpar et al., 2004; Paganelli et al., 2001; Sun et al., 2007). The main drawback of these methods is that the battery life extension is neglected in the proposed control strategy, which is only based on reduction of emission or fuel economy, or improving efficiency.

In this paper, a novel fuzzy rule-based control strategy is proposed, in which two special features are embed- 
ded: predictive control and durability extension of the battery life. Based on the well known load-leveling approach (Hochgraph et al., 2000), the predictive algorithm is designed such that the future path information of the vehicle is also taken into account for generating the control signals. Global positioning systems (GPS) can acquire the knowledge of the obstacles that will be faced in near future, such as heavy traffic, or a steep grade (Rajagopalan et al., 2003; Ichikawa et al. 2004). A fuzzy logic controller is used to generate the control signal based on the predicted future situation of the vehicle. The second feature of the proposed controller is to extend the battery life. Durability of the battery is a crucial problem in hybrid electric vehicles (Hajimiri \& Salmasi, 2007). The State Of Health is the most important parameter describing the life time of a battery, which is related to its power capacity. The most important factors affecting $\mathrm{SOH}$ are battery pick current, number of battery charge/discharge cycles and temperature (Bhangu et al., 2005). Thus, since sustaining the SOH of the battery in a drive-train is critical, the proposed energy management strategy reacts by limiting the charge/discharge cycles, if the $\mathrm{SOH}$ of the drive train battery degrades. Furthermore, in the proposed system, the battery local controller/charger determines the optimal charging profile to limit the SOH degradation, peak current and charging time. In order to verify the performance of the proposed energy management, the algorithms are implemented in ADvance VehcIle SimulatOR (Burch et al., 1999) and compared with conventional power follower rule based method for a series hybrid vehicle. In section 3, by introducing a new cost function, the optimal charging current profile can be obtained in order to decrease charging time and improve battery lifetime. In the local battery controller, an optimization technique such as steepest decent or Genetic Algorithms can be used to determine the charging profile by minimization of the proposed cost function. The optimal charging current is compared with a constant current charging mode for two similar battery cells, which verifies the superiority of the optimal profile.

\section{PREDICTIVE AND PROTECTIVE CONTROL STRATEGY}

This section describes a fuzzy logic based control strategy which utilized the future state of the vehicle and state of the health of the battery for its decision making process. The core of the main controller is the wellknown power follower algorithm. In this algorithm, the engine/generator is operated intermittently to avoid operating in the low output range with poor efficiency or high emissions. If the State of Charge of the battery is at or close to a high limit, the battery provides required power by the electric motor for traction. If the SOC of the battery is within an acceptable range, the EM power is provided by $\mathrm{E} / \mathrm{G}$, while the engine operating point is set by the local engine controller to minimize emission or maximize efficiency. Furthermore, while the battery SOC falls below the lower limit, the engine/generator provides additional power to recharge the battery, while operating in a high efficiency or low emission region, governed by the local engine controller. The battery is also charged by the traction motor in generating mode while braking.

\subsection{Predictive controller}

In order to improve the performance of the above energy management strategy, a predictive controller is augmented. Then, the durability extension of battery life will be taken into account to develop predictive and protective control. A Fuzzy Logic Controller is utilized to generate the control signals based on the predicted future situation of the vehicle. The controller has two inputs and one output. The first input is the difference between the predicted future speed and the present measured speed of the vehicle. The second is the difference of the elevation of the future and the present vehicle position. The output is a control signal which manipulates the recharge and discharge of the battery.

To determine the future state of the vehicle, a time interval of the vehicle's future path is chosen to obtain elevation and traffic. As shown in Figure 2, the controller takes some samples from the elevation and the speed in the selected interval. The samples are averaged to determine the speed and the elevation, as estimates of the future state of the vehicle. For example, if $\mathrm{N}$ samples are taken from the future interval then we have,

Future state $=\frac{\sum_{k=1}^{N}(\text { sampled value })_{i}}{N}$

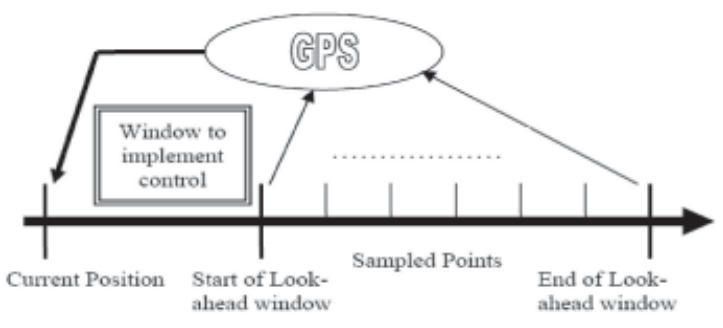

Fig. 2 A sample look-ahead window for predictive control

Essentially, estimation of the future state is halted before the vehicle reaches to a certain point. In fact, in the vicinity of this point, the future state of the vehicle is not estimated.

The fuzzy rule-base is designed to determine how a ve- 
Table 1 Fuzzy rule bases of predictive control strategy

\begin{tabular}{|l|l|l|l|}
\hline $\begin{array}{l}\text { Future } \\
\text { State }\end{array}$ & $\begin{array}{l}\text { Increasing } \\
\text { elevation }\end{array}$ & $\begin{array}{l}\text { Constant } \\
\text { elevation }\end{array}$ & $\begin{array}{l}\text { Decrea sing } \\
\text { elevation }\end{array}$ \\
\hline $\begin{array}{l}\text { Increasing } \\
\text { traffic flow }\end{array}$ & Nothing & $\begin{array}{l}\text { Normal } \\
\text { discharging }\end{array}$ & $\begin{array}{l}\text { High } \\
\text { discharging }\end{array}$ \\
\hline $\begin{array}{l}\text { Constant } \\
\text { traffic flow }\end{array}$ & $\begin{array}{l}\text { Normal } \\
\text { charging }\end{array}$ & Nothing & $\begin{array}{l}\text { Normal } \\
\text { discharging }\end{array}$ \\
\hline $\begin{array}{l}\text { Decreasing } \\
\text { traffic flow }\end{array}$ & $\begin{array}{l}\text { High } \\
\text { charging }\end{array}$ & $\begin{array}{l}\text { Normal } \\
\text { charging }\end{array}$ & Nothing \\
\hline
\end{tabular}

hicle should react to the future states. Fuzzy rules are listed in Table 1. As an example, when the GPS indicates "Decreasing elevation" and "Increasing traffic flow" for the future state, the output command is "high discharging”. In this case, more battery energy is consumed in slower traffic and higher elevation; while the future state of the vehicle, i.e. decreasing elevation and increasing traffic flow, will compensate the high rate of discharging at present.

For each input, three trapezoidal membership functions are assigned, giving a total of nine rules. Linguistic variables assigned to the first input, i.e. the difference between the predicted future speed and the present measured speed of the vehicle correspond to the decrease, constancy and increase of the flow of traffic. For the change in elevation, membership functions of the three inputs correspond to the decrease, constancy and increase of the elevation. The output is assigned with five different crisp values $(-1,-0.5,0,0.5,1)$, ranging from “- 1 " corresponding to high discharging to " 1 " corresponding to high charging.

The degree of consistency between the given facts and the antecedent of each rule is determined in terms of the minimum height of the associated inputs. For the output inference, Center of Area Method which is sometimes called Center of Gravity Method or Centroid Method is used. The formula of the defuzzified value, $d_{C A}(O)$, in which $O$ is the function of degree of consistency defined on a finite universal set $\left\{z_{1}, z_{2}, \ldots, z_{n}\right\}$, is given by

$$
d_{C A}(O)=\frac{\sum_{k=1}^{n} O\left(z_{k}\right) z_{k}}{\sum_{k=1}^{n} O\left(z_{k}\right)}
$$

In this case, the output has five values $(-1,-0.5,0,0.5$, 1 ), hence, $n$ is equal to five. The inferred output is scaled to match "- 1 ”, corresponding to maximum possible discharge power of battery and “ 1 ”, corresponding to maximum possible charge power of the battery. Another parameter included in the proposed control strategy is the upper and lower limits of state-of-charge (SOC) of the battery. For example, if SOC is close to a preset upper bound, charging command of the predictive controller will be bypassed. On the other hand, we have "nothing" command in our fuzzy rule base and it indicates that no difference exists between the future and the present of vehicle situation. Under this circumstance, the proposed algorithm behaves the same as the conventional power follower algorithm.

The proposed predictive algorithm is implemented in ADVISOR software for a series HEV with the specifications listed in Table 2. In order to simulate predictive algorithm, the future speed of the vehicle, determined from the provided drive cycle, is used to predict the traffic flow. Simulation results are compared with the conventional Power Follower Algorithm. The drive-cycle employed in this study is a combination of two urban dynamometer driving schedule (UDDS) and the grade profile is chosen as shown in Figure 3.

Table 2 Components of series traction system

\begin{tabular}{|l|l|}
\hline Generator & Synchronous type, 50KW \\
\hline Traction Motor & Induction type, 75KW, 230v \\
\hline Battery & VRLA, 25 cells, 25 Ah C/5 \\
\hline Internal Combustion Engine & $45 \mathrm{KW}$ \\
\hline
\end{tabular}

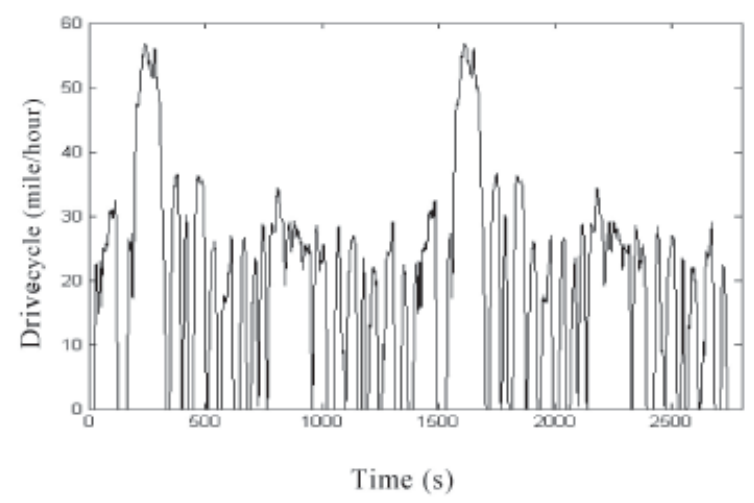

(a) The drive-cycle

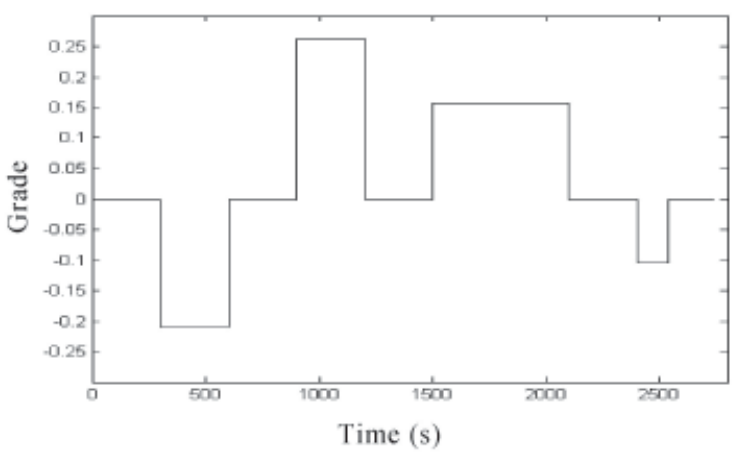

(b) The Grade of the path

Fig. 3 Employed drive cycle and grade for simulation 
The comparison results of the predictive algorithm and PFA are shown in Figure 4. It can be observed that the predictive algorithm is able to track the chosen drivecycle; however, conventional power flower strategy lacks the same performance in some parts of drive-cycle. As shown in Figure 4 (c), the difference between the SOC of the battery in predictive algorithm and PFA becomes more significant in two intervals. One of them starts at 600th second and ends at 900th. The other one starts at 1900th and ends at 2100th. These two intervals are the positions that according to the future state of the vehicle, the algorithm commands higher charge and discharge of the battery.

The fuel consumption and emission of both algorithms are listed in Table 3. It can be observed that the predictive controller improves the fuel economy and different kinds of emissions.

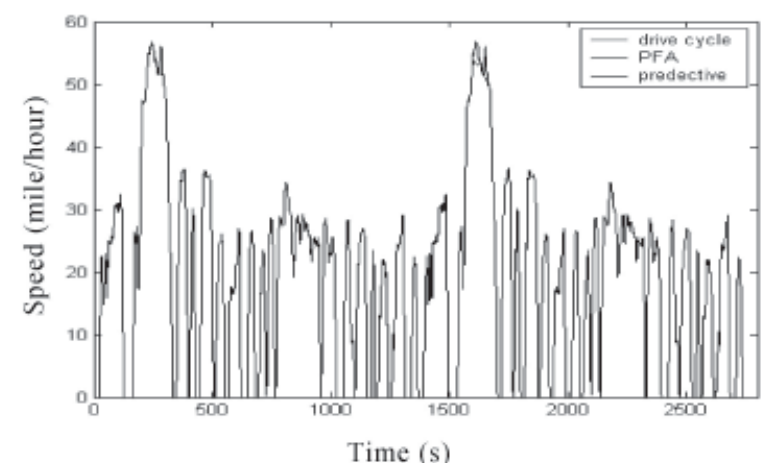

(a) The vehicle's speed profile

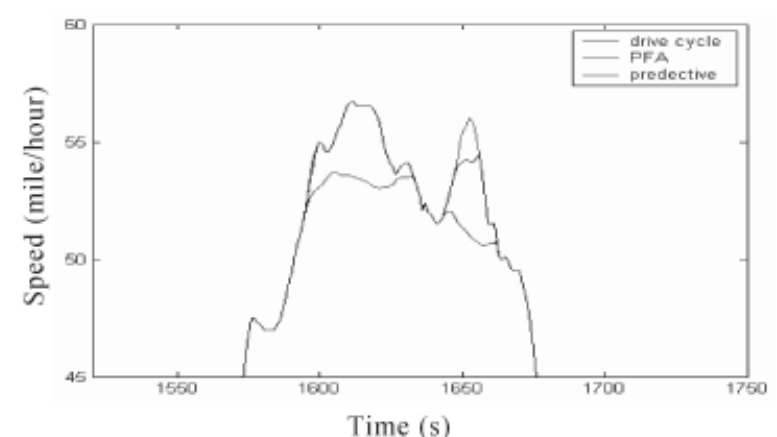

(b) A part of the vehicle's speed profile

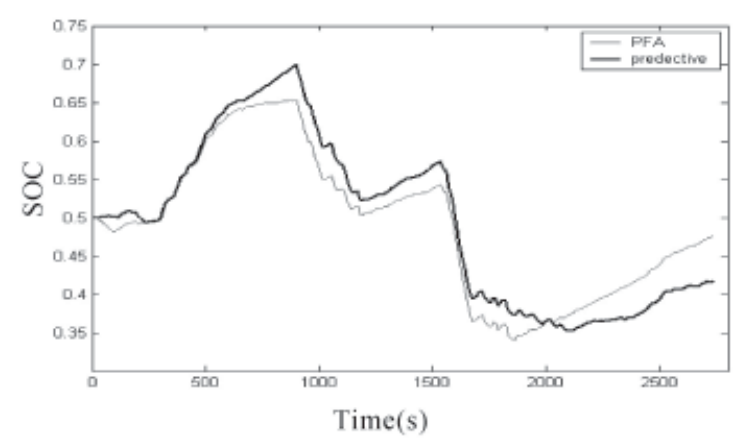

(c) Comparison of Battery's SOC

Fig. 4 Comparison of the predictive and power follower algorithms
Table 3 Fuel consumption and emissions of predictive algorithm, PFA and PPA

\begin{tabular}{|c|c|c|c|}
\cline { 2 - 4 } \multicolumn{1}{c|}{} & \multicolumn{3}{c|}{ Control strategy } \\
\cline { 2 - 4 } \multicolumn{1}{c|}{} & PFA & $\begin{array}{c}\text { Predictive } \\
\text { Algorithm }\end{array}$ & PPA \\
\hline $\begin{array}{c}\text { Fuel } \\
\text { Consumption } \\
{[\mathrm{Lit} / \mathrm{mile}]}\end{array}$ & 0.202 & 0.189 & 0.210 \\
\hline CO $[\mathrm{g} / \mathrm{mile}]$ & 5.080 & 4.293 & 5.340 \\
\hline HC $[\mathrm{g} / \mathrm{mile}]$ & 0.676 & 0.656 & 0.695 \\
\hline NOx $[\mathrm{g} / \mathrm{mile}]$ & 0.894 & 0.878 & 0.911 \\
\hline
\end{tabular}

\subsection{Protective controller}

Based on the predictive and power follower controller, a more inclusive algorithm is suggested such that the battery's $\mathrm{SOH}$ is also taken into consideration, which is entitled as predictive and protective algorithm (PPA). Block diagram of the whole energy management strategy is shown in Figure 5. The state of the health (SOH) is defined as the ability of a cell to store energy, source and sink high currents, and retain charge over extended periods, relative to its initial or nominal capabilities. The available charge stored within a fully charged cell is expected to decrease with cell usage, as active material on the cell plates gradually degrades by mechanisms such as loss of plate active surface area due to repeated dissolution and re-crystallization, loss of electrical contact between metallic grids and active materials, and growth of large inactive crystals of lead sulphate (Bhangu et al.,

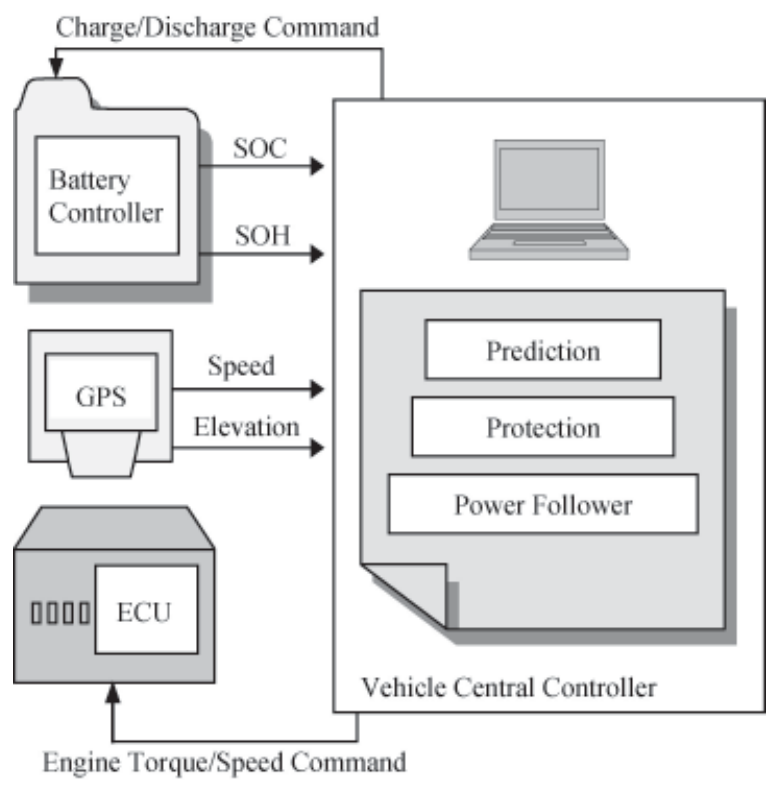

Fig. 5 Block diagram of the proposed predictive and protective energy management system 
2005).

In PPA strategy, the associated fuzzy rule base has three inputs and one output. The first two inputs defined the same as the predictive controller. The third input is the state-of-health of the battery. Thus, in this case the control signal is generated based on the battery health condition, in addition to the future state of the vehicle. When $\mathrm{SOH}$ of battery is in a critical condition, the controller limits the control signal which manipulates recharge and discharge of the battery. This results in the reduction of number of battery charge/discharge cycles during an interval. On the other hand, when the algorithm limits the battery power, the engine should generate more power, so the emission and fuel consumption increase. This means that the algorithm sacrifice the fuel economy and emissions, in order to extend the battery life, which is unavoidable sometimes to save the battery life.

In order to simulate the proposed PPA in ADVISOR, the $\mathrm{SOH}$ of the battery is estimated, as explained in the next section, normalized between 0 and 1 and fed to the controller. Here, " 1 " corresponds to the best condition of battery life and " 0 " corresponds to the condition in which battery is almost dead. The fuzzy rule base in predictive and protective algorithm is designed to determine how a vehicle should react based on the battery's health condition and future state of the vehicle. The table of fuzzy rules for the first two inputs, the difference of speed and difference of elevation, are the same as Table 2. Nevertheless, depending on the condition of SOH input, we have different definition for "high charging", "charging", "high discharging” and "discharging" commands. Each input is assigned with three trapezoidal membership functions, giving a total of 27 rules. For $\mathrm{SOH}$, the first membership function corresponds to good condition for battery life, the second one corresponds to not critical condition and the last one corresponds to critical condition. The output has nine different crisp values $(-1,-0.7,-0.5,-0.3,0,0.30 .5,0.7,1)$. The numbers " -1 ", “- 0.5 ", " 0 ", " 0.5 " and " 1 " correspond to high discharging, discharging, nothing, charging and high discharging for good condition of $\mathrm{SOH}$, respectively. Besides, the values “-0.7”, “-0.3”, “0”, “0.3”, “0.7” correspond to high discharging, discharging, nothing, charging and high discharging for not critical condition of SOH, respectively, and " 0 " corresponds to all commands of critical condition of $\mathrm{SOH}$.

The proposed predictive and protective algorithm is implemented in ADVISOR for the same series HEV and the results are compared with the proposed predictive controller. The drive-cycle and the grade profile used in this test are the same as before (Figure 3). The simulation results based on PPA are shown in Figure 6. As Shown in Figure 6 (b), while the SOH of the battery is

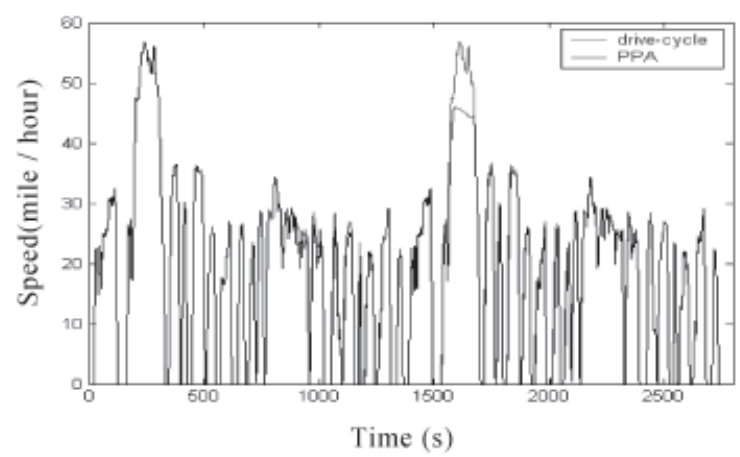

(a) Drive-cycle and predicted vehicle speed based on PPA

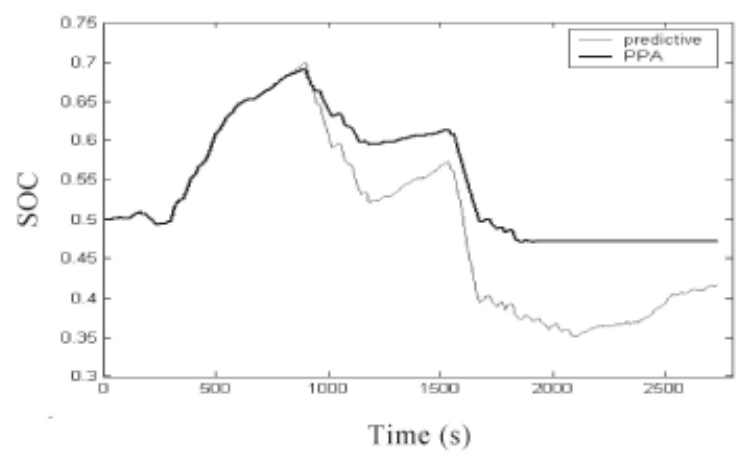

(b) Comparison of battery's SOC based on predictive algorithm and PPA

Fig. 6 Simulation results for protective and predictive algorithm

in good condition, the SOC of the battery in the new proposed algorithm matches the SOC obtained from the predictive algorithm. After degradation of the $\mathrm{SOH}$ condition, charging and discharging of battery is restricted in the PPA algorithm. If $\mathrm{SOH}$ condition becomes critical, charging and discharging of the battery completely stops to protect battery from serious damages. Nevertheless, it can be observed that sometimes, PPA may not trace the drive-cycle exactly, as opposed to predictive strategy. Furthermore, fuel consumption and emissions associated with these strategies are shown in Table 3. It can be observed that in comparison with the two other methods, in PPA strategy, the fuel economy has been decreased different kinds of emissions are increased. This is the price that we have to pay for battery protection sometimes. In other words, based on PPA, it is worthy to burn more fuel sometimes to extend battery life. This is a logical decision considering the battery expenses in series hybrid vehicles.

\section{CHARGE OPTIMIZATION CONTROL}

Not only can the central controller increase the battery life expectation based on an appropriate energy management strategy, but also the battery local charger/controller is able to improve battery's state of health and also the charging time, by optimization of the charging profile. To achieve this goal and also to estimate battery's 


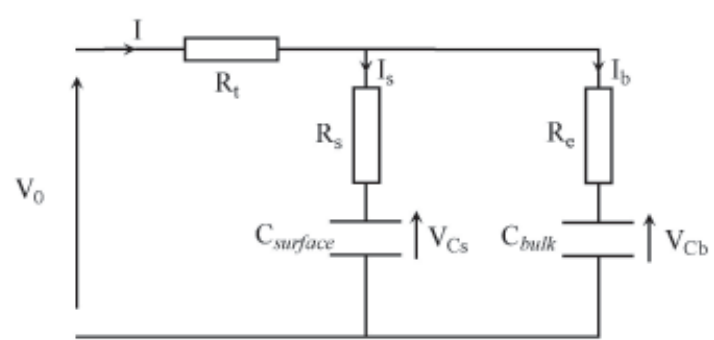

Fig. 7 VRLA battery model

$\mathrm{SOH}$, which is fed back to the central controller, a battey model is used, as shown in Figure 7. This model consists of a bulk capacitor $\mathrm{C}_{\text {bulk }}$ to characterize the ability of the battery to store charge, a capacitor to model surface capacitance and diffusion effects within the cell $\mathrm{C}_{\text {surface}}$, a terminal resistance $\mathrm{R}_{\mathrm{t}}$, surface resistance $\mathrm{R}_{\mathrm{s}}$ and end resistance $\mathrm{R}_{\mathrm{e}}$. The ability of the battery to store energy, in other words battery's state of health, decreases with cell usage. This means that the bulk capacitor, $\mathrm{C}_{\text {bulk}}$, is not constant and deceases over time. Besides, by choosing $\mathrm{x}=[\mathrm{Vbs}, \mathrm{Vcs}$, Vo, Cbulk] as the state vector, the state equations of the battey is obtained in the form of $d x / d t=f(x, u)$ (Bhangu et al., 2005).

To determine the optimum charging profile, the following cost function is minimized based on the above model

$J=\int_{0}^{t_{t}}\left\{\left(V_{C b_{\max }}-V_{C b}\right)^{2} Q_{1}+\left(C_{b, \max }-C_{b u l k}\right)^{2}+I^{2} R\right\} d t$,

where $\mathrm{V}_{\mathrm{cb} \text {,max }}$ is the voltage at the end of the charging process, and $\mathrm{C}_{\mathrm{b} \text {,max }}$ stands for the maximum battery capacity at the beginning of the charging process. Minimization of the first term results in the charging time optimization, while minimizing the second and the third terms restricts battery's SOH degradation. The optimal charging profile is obtained based on the minimization of the cost function for the aforementioned battery model. In order to feedback $\mathrm{SOH}$ to the central controller, $\mathrm{C}_{\text {bulk }}$ is estimated as a criterion of the state of health of the battery by the Extended Kalman Filter, as a most widely used methods for state estimation of non-linear systems (Plett, 2004). Once $\mathrm{C}_{\text {bulk }}$ is estimated, the normalized $\mathrm{SOH}$ is determined as $\mathrm{SOH}_{\text {norm }}=\mathrm{C}_{\mathrm{bulk}} / \mathrm{C}_{\mathrm{b}, \max }$.

In order to verify the proposed charging algorithm, two VRLA 2Ah battery cells, are tested. The batteries are charged with the constant charging and optimized charging profile. Then, they are discharged through resistive load to $20 \%$ of the initial charge. This cycle was repeated 75 times. At the end of the tests, their $\mathrm{SOH}$ was estimated. The normalized estimated $\mathrm{SOH}$ parameter for the battery charged with constant current profile was about $6 \%$ less than the one charged with optimized profile. Besides, the average charging time for the optimal profile was $11 \%$ less than the other one. It can be real- ized that optimized charging profile improves state of health of the battery and reduces charging time, which is very important for safety and economical reasons.

\section{CONCLUSION}

In this paper, a modified power follower energy management system for series hybrid electric vehicles is proposed, which is based on protection of the vehicle's battery and prediction of the future vehicles' path. First, a fuzzy predictive algorithm is integrated into a conventional power follower such that the future path information of the vehicle is taken into account for generation of the control signals. The simulation results show that for areas with variable road elevation, the predictive version has better tracing capability. Then, the energy management system is augmented with a control block to increase the state of health (SOH) of the power train battery. This block may restrict battery charging and discharging depending in the battery's SOH. This approach may increase the emissions sometimes to extend battery life, especially while it is in a critical condition. Considering the high cost of battery cells in hybrid vehicles, it seems to be a logical decision. Besides, the optimal charging current can be obtained, in order to decrease charging time and improve battery lifetime, by minimization of a new cost function. The main point is that in hybrid electric vehicles, an advanced energy management strategy should consider emission reduction along with prolonging of battery's life duration.

\section{References}

Burch, S., M. Cuddy, and T. Markel, Advisor 2.1 Documentation, National Renewable Laboratory, 1999.

Bhangu, B. S., P. Bentley, D. A. Stone, and C. M. Bingham, Nonlinear Observers for Predicting Stateof-Charge and State-of-Health of Lead-Acid Batteries for Hybrid-Electric Vehicles, IEEE Transactions on Vehicular Technology, Vol. 54, No. 3, 2005.

Chan, C. C., The state of the art of electric and hybrid vehicles, Proceedings of the IEEE, Vol. 90, No. 2, 247-275, 2002.

Delpar, S., J. Lauber, T. M. Guerra, and J. Rimaux, Control of a parallel hybrid powertrain: optimal control, IEEE Transactions on Vehicular Technology, Vol. 53, No. 3, 872-880, 2004.

Ehsani, M., Y. Gao, S. E. Gay, and A. Emadi, Modern Electric, Hybrid Electric, and Fuel Cell Vehicles, CRC Press LLT, 2005.

Hajimiri, M. H., and F. R. Salmasi, A Fuzzy Energy Management Strategy for Series Hybrid Electric Vehicle with Predictive Control and Durability Extension of the Battery, Proceedings of IEEE Hybrid Electric Vehcile Conference, New Delhi, India, 2007. 
Hochgraph, C. G., M. J. Ryan, and H. L. Wiegman, Engine control strategy for a series hybrid electric vehicle incorporating load leveling and computer controlled energy management, SAE J. SAE/SP-96/1156, 11-24, 2000.

Ichikawa, S., Y. Yokoi, S. Doki, S. Okuma, T. Naitou, T. Shiimado, and N. Miki, Novel energy management system for hybrid electric vehicles utilizing car navigation over a commuting route, Proceedings of IEEE Intelligent Vehicles Symposium, 2004.

Miller, J. M., Propulsion Systems for Hybrid Vehicles, IEE Power and Energy Series, 2004.

Paganelli, G., S. Delpart, T. M. Guerra, J. Rimaux, J. J. Santin, Equivalent Consumption minimization strategy for parallel hybrid powertrains, Proceedings of Fall VTC-01 Conference Sponsored by VTS and IEEE, 2001.

Plett, G. L., Extended Kalman filtering for battery management of LiPB-based HEV battery packs Part 3. State and parameter estimation, Journal of Power Sources, Vol. 134, 277-292, 2004.

Rajagopalan, A., G. Washington, G. Rizzoni, and Y. Guezennec, Development of Fuzzy Logic and Neural Network Control and Advanced Emissions Modeling for Parallel Hybrid Vehicles, National Renewable Energy Laboratory, NREL/SR-540-32919, 2003.

Salmasi, F. R., Control Strategies for Hybrid Electric Vehicles: Evolution, Classifiction, Comparison and Future Trends, IEEE Transaction on Vehicular Technology, Vol. 56, No. 5, 2393-2404, 2007.

Schouten, N. J., M. A. Salman, and N. A. Kheir, Fuzzy Logic Control for Parallel Hybrid Vehicles, IEEE Transaction Control System Technology, Vol. 10, 460468, 2002.

Sun, H., J. -H. Jiang, X. Wang, Optimal Torque Management Strategy For A Parallel Hydraulic Hybrid Vehicle, International Journal of Automotive Technology, Vol. 8, No. 6, 791-798, 2007.

(Received September 27, 2008; accepted November 1, 2008) 